\title{
Les relations conifères-scolytides : importance et perspectives de recherches
}

\author{
F. LIEUTIER et J. LEVIEUX \\ I.N.R.A., Station de Zoologie forestière, Centre de Recherches dorléans \\ Ardon, F 45160 Olivet
}

\begin{abstract}
Résumé
Les possibilités de dégâts des scolytides dans les peuplements forestiers résineux résultent de deux facteurs complémentaires, d'une part l'existence d'arbres affaiblis sous l'effet d'un stress, d'autre part un certain niveau de population de ravageurs. Une présentation rapide de l'état actuel des recherches dans ces domaines est effectuée d'après les travaux nord-américains et quelques travaux européens, en insistant sur ceux qui accordent un rôle important aux réactions de défense de l'arbre. Des orientations de recherches sont ensuite suggérées pour la France. La démarche générale proposée est, à court terme, d'apprécier dans quelle mesure et pour quelles espèces le «modèle américain» est transposable en France; certains scolytides européens d'intérêt économique possèdent, en effet, une position systématique et une biologie assez différentes des espèces nord-américaines ayant servi à l'élaboration du modèle. A plus long terme, on pourrait viser, d'une part la compréhension des modalités d'installation des espèces françaises pour pouvoir proposer à l'améliorateur des bases complémentaires de sélection, d'autre part la mise au point d'indices permettant d'estimer les effectifs de ravageurs. Les thèmes de recherche proposés sont : étude fine du mécanisne des perturbations physiologiques provoquées par le stress hydrique ; mise au point de critères permettant d'apprécier l'état de délicience des arbres; rôle des champignons associés dans les mécanismes d'installation des espèces françaises; dispersion et répartition des insectes en phase endémique ; mise au point d'une méthode d'échantillonnage des populations au niveau parcellaire; étude des facteurs responsables des variations des niveaux de population.
\end{abstract}

Le forestier européen sait depuis longtemps que les scolytes, à l'état endémique, sont des ravageurs de faiblesse, c'est-à-dire qu'ils ne réussissent généralement leur installation que sur des arbres déficients. Cette observation commune en Europe ressort également des nombreux travaux effectués en Amérique du Nord dans les immenses forêts de conifères, là où les essences en place sont d'origine. Avec des populations épidémiques toutefois, les arbres en parfaite santé eux-mêmes peuvent succomber aux attaques alors denses et répétées des scolytides. En outre, même sur les arbres affaiblis, les attaques isolées aboutissent rarement et le succès de l'installation nécessite le plus souvent une quantité minimale d'attaquants (setiil de population).

Il apparaît donc que les possibilités de dégâts dans les peuplements forestiers résultent de deux facteurs complémentaires essentiels, d'une part l'existence d'arbres 
réceptifs, c'est-à-dire affaiblis sous l'effet d'un stress, d'autre part un certain niveau de population de ravageurs. Pour espérer déboucher sur la compréhension des mécanismes de déclenchement des attaques, compréhension qui devrait permettre d'élaborer une méthode de lutte préventive, les recherches doivent s'orienter vers ces deux aspects.

Nous nous proposons dans ce qui suit d'effectuer le point sur l'état actuel des recherches dans ces domaines pour les scolytides des conifères en général, d'après les travaux nord-américains et quelques travaux européens, puis nous exposerons nos idées sur la façon d'aborder les problèmes français. Nous précisons néanmoins, tout de suite, qu'il nous paraît absolument essentiel de ne pas oublier que l'arbre est un organisme vivant, ce qui signifie qu'il n'est jamais passif à une agression, quelle qu'elle soit et que, même affaibli, il doit être capable dans certaines limites sans doute, d'élaborer un processus de défense. C'est pourquoi, lors de cette mise au point, nous attacherons une attention particulière aux travaux en accord avec cette conception.

\section{Les arbres réceptifs et les relations conifères-scolytes}

L'installation des scolyies sur les arbres s'effectue en plusieurs phases successives. L'attaque commence par l'arrivée de quelques insectes pionniers qui « choisissent $"$ les sujets susceptibles de convenir à leur installation. Un mélange de substances volatiles émises par l'arbre et par les pionniers confère alors à l'ensemble arbre + insectes une très grande attractivité et détermine l'agrégation massive d'une très grande quantité d'insectes sur l'hôte choisi. Cette agrégation est en outre accrue considérablement par le fait que les insectes attirés contribuent eux-mêmes, dès leur arrivée, à augmenter l'attractivité du milieu. On a donné le nom de «phéromones d'agrégation » aux médiateurs chimiques responsables de ce phénomène. La troisième et dernière phase correspond à l'installation définitive des insectes; celle-ci implique la mort de l'arbre dans la quasi totalité des cas et conditionne toujours la survie de la descendance du scolytide. Sa l'éussite est déterminée par les capacités de défense du végétal.

\subsection{Le choix des arbres par les insectes-pionniers}

Les modalités de ce choix ne sont pas encore clairement comprises et semblent dépendre des espèces en cause. Dans certains cas au moins, le spectre d'odeur de l'arbre formé par différents constituants des résines subirait une altération sous l'effet d'un stress hydrique (Chararas, 1959 a, 1979; Pesson \& Chararas, 1969). Les produits d'oxydation des terpènes (attractifs pour les insectes) seraient en quantité plus élevée dans le spectre d'odeur des arbres déficients, tandis que les terpènes, en général répulsirs à haute concentration, scraient présents en quantités plus faibles. RAFFA \& BERRYMAN (1983 a) signalent aussi que la quantité de terpènes oxydés est plus grande chez les individus de Pinus contorta susceptibles aux attaques que chez les individus résistants. Dans d'autres cas, le choix des arbres par les pionniers s'effectuerait au hasard, les capacités de défense de l'arbre et les stimuli gustatifs déterminant finalement l'acceptation ou le rejet de l'hôte (HYNUM \& BERRYMAN, 1980 ; Monck ct coll., 1981 ; RafFa \& Berryman, 1982 a ; WoOn, 1982). Par ailleurs, dans 
tous les cas, il est probable que la vision intervient dans l'attraction à distance. Les scolytides sont en effet attirés par des silhouettes linéaires verticales (ScHONHERR, 1977 entre autres); cela ne leur permet sans doute pas de choisir préférentiellement les arbres déficients, mais on ne connaît pas le rôle de la vision des couleurs dans l'orientation de ces insectes.

\subsection{L'agrégation}

Les médiateurs chimiques d'agrégation des scolytides ont fait l'objet d'importantes recherches ces quinze dernières années. De nombreuses substances ont été isolées et synthétisées. C'est toujours un mélange de plusieurs produits qui est actif et leur mode d'action et leur fonction apparaissent complexes, dépendant d'un grand nombre de facteurs tels que l'isomérie optique, la concentration des substances ou la nature des mélanges dans lesquels elles se trouvent. Des interactions multiples existent entre les différents composés, ce qui permet des communications entre scolytides d'espèces différentes. En outre, la stridulation par l'un, l'autre ou les deux sexes interfère avec l'émission des phéromones. On trouvera de nombreuses précisions sur les phéromones d'agrégation des scolytides dans les publications de SILVERSTEIN (1977) et de WoOD (1982).

Une caractéristique fondamentale de ces médiateurs chimiques est qu'ils ne sont généralement pas des sécrétions des scolytides, mais, au contraire, des substances d'origine végétale modifiées par passage dans le tube digestif des insectes. Ainsi, la plupart des substances isolées chez les scolytides des conifères dérivent, par transformation chimique simple, des terpènes de l'hôte. Mêmes s'ils ne sont sans doute pas seuls responsables, les microorganismes symbiotiques du tube digestif paraissent jouer un rôle fondamental dans cette transformation (BRAND et coll., 1975; CHARARAS et coll., 1979).

Il est certain que les recherches sur les phéromones d'agrégation des scolytides ont apporté des informations extrêmement précieuses sur le comportement de ces insectes et leurs possibilités de colonisation des arbres, mais elles ont souvent considéré ces derniers comme un support inactif. Il est très important, au contraire, de considérer l'arbre comme un être vivant. Ce fait apparaît au niveau du choix effectué par les insectes pionniers et, comme nous le verrons, dans les mécanismes conditionnant l'installation définitive des ravageurs. Il n'est certainement pas non plus à négliger pendant le déroulement de la phase d'agrégation. RAFFA \& BERRYMAN $(1983 \mathrm{~b})$ ont en effet montré, lors des attaques de Pinus contorta par Dendroctonus ponderosae, que la durée de la phase d'agrégation était directement liée à la vigueur du végétal et que l'agrégation elle-même semblait pouvoir être inhibée par l'intensité de la réaction de défense de certains arbres (cf. infra).

\subsection{L'installation définitive des insectes et les réactions de défense de l'arbre}

On peut, avec BERRYMAN (1972), considérer que les conifères disposent de deux systèmes de défense vis-à-vis des attaques de scolytes :

- la réaction primaire de défense correspond à un écoulement de résines provoqué par l'action traumatique de l'insecte. C'est une réaction rapide, passive, due, soit à la section de canaux résinifères préexistants (pin, épicéa, mélèze), soit à la 
néoformation de poches ou de canaux à résine chez les arbres qui n'en possédaient pas déjà (sapin, cèdre, ...). Ces résines primaires sont sécrétées lors de n'importe quelle blessure entamant l'intégrité des tissus conducteurs. Lors des attaques de scolytes, les résines primaires, quand elles sont en quantité importante, peuvenî empêcher l'insecte de pénétrer dans l'arbre, soit qu'il se noie ou s'englue, soit qu'il s'échappe et cherche un autre arbre plus propice à son installation. Certains insectes ont cependant la possibilité de forer leur trou de pénétration à travers le flux de résines. Les cônes de résine percés d'une galerie que l'on observe en surface des arbres attaqués par Tomicus piniperda ou Dendroctonus micans par exemple sont la manifestation de cette lutte entre l'insecte et les résines primaires. La composition chimique des résines primaires, en particulier les terpènes qu'elles contiennent, pourrait aussi constituer un mécanisme de dissuasion vis-à-vis de l'insecte. Par ailleurs, il semble que chez certains arbres, l'intensité de la réaction primaire puisse inhiber l'agrégation, en limitant l'émission des phéromones par les insectes arrivés sur l'arbre, ou en gênant la perception de ces substances par les insectes en vol, par suite, par excmple, d'une trop grande richesse en terpènes (RAFFA \& BERRYMAN, 1983 b). La réaction primaire de défense a donc un rôle important lors des deux premières phases d'installation des scolytes;

- la réaction secondaire est dirigée contre les champignons transportés par les insectes; on sait, en effet, que la plupart des scolytides sous-corticoles transportent dans des organes variés les spores de divers champignons (Graham, 1967 ; FranckeGrossman, 1967). Les interactions entre l'arbre et ces derniers ont été particulièrement étudićes au Canada et aux Etats-Unis dans le cas de l'installation de Dendroctonus ponderosae sur Pinus contorta ou de Scolytus ventralis sur Abies grandis (REID et coll., 1967 ; Berryman, 1972 ; Shrimpton, 1973, 1978 ; SaFranytK et coll., 1975 ; Russel \& Berryman, 1976 ; Wong \& Berryman, 1977 ; RafFa \& Berryman, 1982 b, 1983 a). L'introduction (artificielle ou par l'insecte) du champignon provoque chez l'arbre une vive réaction autour du point d'inoculation. En avant du front de développement du pathogène, les cellules parenchymateuses se nécrosent et synthétisent des quantités importantes de résines secondaires. Les tissus parenchymateux imprégnés par ces résines deviennent alors inutilisables par le champignon et incompatibles avec le forage d'unc galeric et la survie des oufs et des larves des scolytes. Ils contiennent des concentrations élevées de divers composés toxiques tels que des monoterpènes et des polyphénols et, après cristallisation des résines possèdent une grande dureté. En outre, quand la réaction est vigoureuse, elle se termine par la crćation d'un bourrelet cicatriciel qui isole le champignon du reste du végétal et lui interdit l'accès aux substances nécessaires à son développement, en particulier aux glucides contenus dans les tissus inaltérés.

Il s'agit donc d'une réaction dynamique, totalement différente de la réaction primaire et correspondant à ce que Klement \& Goodman (1967) ont défini comme une réaction d'hypersensibilité chez les végétaux. L'intensité de cette réaction, qui intéresse à la fois les insectes pionniers et ceux qui sont attirés par les phéromones, conditionnerait directement la réussite de l'installation définitive des insectes ( $3^{\mathbf{e}}$ phase). Le rôle du champignon scrait de vaincre la réaction de l'arbre et d'affaiblir sa résistance favorisant ainsi l'installation définitive du scolyte et la survie de sa descendance. Le résultat dépendrait donc d'une part du rapport de force champignonarbre, d'autre part, de la possibilité qu'aurait l'insecte, par son activité de forage, de prendre de vitesse la réaction secondaire de défense déclenchée par la présence du champignon dans l'arbre. Si l'arbre ne réagit pas assez rapidement et vigoureu- 
sement, le champignon se développe, l'installation du scolyte réussit et sa descendance survit. Si, au contraire, l'arbre est suffisamment vigoureux pour contenir le développement du champignon ef l'avancée de l'insecte, l'attaque du scolyte avorte.

\section{Les niveaux de population des scolytides}

Dans le processus d'installation décrit plus haut, les relations entre les capacités de défense des arbres et l'agressivité des champignons apparaissent primordiales. Or, la synthèse des résines secondaires et, en particulier, des terpènes qu'elles ren ferment est grande consommatrice d'énergie; la biosynthèse d'une molécule de monoterpènes par exemple correspond pour l'arbre à une perte potentielle de 90 molécules d'A.T.P. (WRight et Coll., 1979). La résistance de l'arbre serait donc conditionnée, directement ou indirectement, par la quantité d'énergie qu'il serait capable de mobiliser rapidement. C'est de ce point de vue que le niveau des populations de scolytides jouerait un rôle fondamental. En effet, selon le schéma précédent, plus le nombre d'attaquants est élevé, plus l'est aussi le nombre de points d'inoculation du champignon. Plus grandes sont alors les chances pour l'agresseur, de provoquer une demande énergétique suffisamment forte pour que l'hôte ne puisse y subvenir et pour que l'installation définitive réussisse. Dans une telle stratégie, on comprend que les attaques réussissent plus facilement, c'est-à-dire avec un nombre d'assaillants plus réduit, sur les arbres déficients, chez lesquels les réserves énergétiques sont diminuées.

Dans ces conditions, le rôle principal des phéromones d'agrégation serait de multiplier rapidement le nombre de points d'inoculation. Il semble exister à ce sujet une étroite corrélation entre la vigueur des arbres et l'émission des phéromones. Ainsi, selon RafFa \& Berryman (1983 b), comme l'insecte utilise les terpènes, composés de défense de l'arbre-hôte, comme précurseurs de ses phéromones, l'émission de ces dernières dure d'autant plus longtemps et les insectes attirés sont d'autant plus nombreux que l'arbre est plus apte à synthétiser des quantités importantes de terpènes. Les arbres se défendant vigoureusement seraient donc assaillis par des quantités élevées d'insectes. Quand la réaction de défense de l'arbre aurait été dominée par le champignon, l'émission de phéromones s'arrêterait d'elle-même.

Les relations entre le niveau des populations de scolytides et la vigueur des arbres sont expliquées de la façon suivante (BERRYMAN, 1976, 1978, 1982 a ; RAFFA \& Berryman, 1980, 1983 b) : pour un bas niveau de population de scolytes, seuls les arbres très déficients et souffreteux sont colonisés avec succès car le seuil critique de densité d'inoculation est pour eux faible. En outre, ces arbres ont peu de liber, le taux de multiplication des insectes y est donc réduit (Amman, 1972; Berryman, 1976). De plus, la nourriture disponible étant rare (peu d'arbres déficients), la mortalité des scolytes pendant la phase de recherche de l'hôte est importante. Les populations se maintiennent ainsi naturellement à l'état endémique.

Le passage à l'épidémie et le déclenchement d'une pullulation pourrait avoir deux origines :

1. Le nombre d'arbres en difficulté physiologique se trouve augmenté de façon importante par suite d'accidents climatiques comme une sécheresse intense et prolongée ou par suite d'une sénescence exagérée des peuplements, par exemple. Ce 
phénomène correspond à un abaissement conséquent du seuil critique d'inoculation de chaque arbre d'où leur colonisation possible, même à bas niveau de population. et donc la multiplication brutale et importante des insectes, favorisée par l'existence, parmi les arbres en difficulté, de nombreux individus à liber épais. Par la suite, même si les conditions redeviennent favorables aux arbres, les populations de scolytes se trouveraient à un tel niveau qu'elles permettraient de toute façon d'atteindre le seuil critique d'inoculation des arbres vigoureux.

2. Le nombre d'insectes se trouve brusquement augmenté grâce à une abondante nourriture immédiatement disponible (cas d'une tempête provoquant d'importants chablis par exemple). Le niveau des populations devient alors tel que des densités d'attaques supérieures au seuil critique d'inoculation des arbres vigourcux sont atteintes.

Dans les deux cas, la multiplication des populations devient possible sur les arbres vigoureux à liber épais. Des seuils de plus en plus élevés sont alors atteints. Le phénomène s'amplifie rapidement de lui-même et pour un très haut niveau de population, même des arbres très vigoureux, disposant de réserves énergétiques importantes, pourraient être vaincus. Ainsi, pourraient s'expliquer l'explosion brutale let la généralisation fréquente des attaques de scolytes, en même temps que leur caractère occasionnel. Il existerait donc un seuil épidémique de population au-delà duquel les processus naturels de régulation ne joueraient plus leur rôle de «feedback » négatif sur la croissance des populations, mais acquerraient, au contraire, un rôle positif favorisant la croissance. Pour BERryman (1982 a), ce phénomène serait une caractéristique des insectes à pullulations occasionnelles. Dans le cas des scolytes, le seuil épidémique correspondrait au niveau de population permettant de dépasser le seuil critique d'inoculation des arbres vigoureux; le processus naturel de régulation le plus important serait la résistance des arbres. Le basculement d'une action de feed-back négatif vers une action positive serait dû à ce que les arbres résistants sont en général ceux qui ont le liber le plus épais.

Toutefois, comme le font remarquer RafFA \& Berryman (1983 b), de telles relations entre populations de ravageurs et arbres sont incompatibles avec la persistance du système hôte-parasite lui-même puisque, en période épidémique et en l'absence d'intervention humaine efficace, elles peuvent théoriquement conduire à la disparition de la totalité des arbres. En fait, le système se maintiendrait d'une part grâce à l'existence d'arbres capables de résister à de très hautes densités d'inoculation (BERRYMAN, $1982 \mathrm{a}$ ), d'autre part parce que certains arbres ne seraient jamais l'objet d'attaques massives (WARING \& PITMAN, 1983). La raison de cette absence d'attaque pourrait être la possibilité d'inhiber l'agrégation (cf. supra). Après la pullulation, les scolytes seraient donc décimés par le manque de nourriture et les populations retourneraient à l'état endémique.

\section{Orientations des recherches en France}

La théorie que nous venons de résumer, expliquant les modalités d'installations des scolytes sur les arbres, en même temps que les mécanismes de régulation des populations de ces insectes et l'existence de leurs pullulations occasionnelles, accorde une extrême importance et une grande généralité au rôle joué par l'arbre lui-même. Chararas $(1962,1979)$ puis Carle (1975) ont déjà souligné, depuis de nombreuses 
années, en France, ce rôle fondamental du végétal, mais l'originalité de la théorie rapportée ci-dessus réside en ce qu'elle accorde une place prépondérante aux réactions de défense de l'arbre. Ce "modèle * a été élaboré à partir de travaux effectués dans leur grande majorité en Amérique du Nord, principalement sur deux espèces de scolytes inconnues en Europe, Dendroctonus ponderosae et Scolytus ventralis. Sur l'ancien continent, les scolytes de grand intérêt économique sont au nombre de cinq, Ips acuminatus, Ips sexdentatus, Ips typographus, Tomicus piniperda et Dendroctonus micans, tous présents en France mais absents sur le nouveau continent. Toutefois, on peut quand même penser que les modalités d'installation des scolytides européens doivent être semblables, dans leurs grandes lignes au moins, à celles des Scolytides d'outre-atlantique. Un des objectifs à court terme des recherches consacrées aux scolytides en France pourrait donc être d'apprécier dans quelle mesure et pour quelles espèces le «modèle américain» est transposable dans notre pays. Ces recherches pourraient viser à plus longue échéance, d'une part la compréhension des modalités d'installation des scolytides français pour pouvoir à terme proposer à l'améliorateur des bases complémentaires de sélection des arbres forestiers, d'autre part la mise au point d'indices permettant d'estimer les effectifs de ravageurs. Nous avons déjà indiqué (Lieutier, 1984) les principales voies de recherches que nous pensons devoir être développées en France; nous les reprendrons ici en précisant quelques points.

\subsection{Les arbres réceptifs et les relations arbres-scolytes; les modalités d'installation des insectes}

Bien que d'origine variée (climatique, édaphique, pathologique, etc.) le stress hydrique est dans la très grande majorité des cas la cause de l'affaiblissement des arbres et se trouve donc presque toujours à l'origine des attaques de scolytes. Une étude fine du mécanisme des perturbations physiologiques provoquées par ce stress serait donc d'une grande utilité. Chararas $(1959 \mathrm{~b}, 1979)$ indique que l'augmentation. de la concentration des sucs cellulaires, sous l'effet de la sécheresse, provoque une élévation importante de la pression osmotique, phénomène qui peut déterminer des troubles physiologiques graves et des lésions cellulaires lorsque les valeurs dépassent. un certain seuil variable selon les essences. D'après cet auteur, l'adaptation d'une essence végétale à la sécheresse serait fonction dans ces conditions de son aptitude à supporter une élévation importante de la pression osmotique. On ne sait pas toutefois quelle est la nature des troubles physiologiques consécutifs à cette élévation de la pression osmotique. Cependant, diverses études ont rapporté des variations importantes de la composition glucidique et des modifications du métabolisme des sucres dans les arbres soumis à un stress hydrique (HobGes \& Lor Io, 1969 ; Hanover, 1975 ; Wright et coll., 1979). Ces voies de recherches sont à développer non seulement au niveau des modifications de la composition glucidique, mais aussi au niveau des mécanismes enzymatiques concernés par les modifications du métabolisme de ces composés.

De telles études, outre une meilleure compréhension des mécanismes de la déficience, pourraient permettre de déboucher sur la mise au point de critères permettant d'apprécier celle-ci. On ne dispose actuellement en France d'aucun moyen de détecter assez tôt les arbres susceptibles aux attaques. La mesure de la pression osmotique utilisée par Craparas $(1959 \mathrm{~b}, 1979)$ dans les pays méditeranéens à longue saison sèche n'est pas transposable en pays tempéré (Chararas, 1974). 11 on 
est de même de la mesure de la pression d'exsudation des oléorésines développée par ViTe (1961) et VITE \& Wood (1961), qui dépend plus du nombre et de la taille des canaux résinifères que d'une sensibilité aux attaques d'insectes (HoDGes \& Lorio, 1971) et qui, de toute façon, ne correspond qu'à une appréciation de l'intensité de la réaction primaire de défense seule. Des recherches sur les échanges hydriques entre le végétal et le milieu environnant (air et sol) pourrait aussi être précieuses pour l'élaboration d'un critère de déficience. La prise en considération de divers coefficients de croissance, ainsi que Waring et Pitman (1980) l'ont fait pour Pinus contorta, en même temps que de diverses caractéristiques morphologiques et stationnelles, pourrait aussi fournir des renseignements utiles sur la vitalité des arbres, en liaison avec les attaques de scolytes. On peut par ailleurs envisager la mise au point d'un test biologique fondé sur l'importance de la réaction d'hypersensibilité à l'introduction d'un champignon véhiculé par un scolyte. Divers essais ont déjà fourni des résultats intéressants (SHRIMPTON \& REID, 1973 ; RAFFA \& BERRYMAN, $1982 \mathrm{c}$ ), mais la lenteur de l'expérimentation et la blessure infligée à l'arbre lors de la lecture des résultats impliquent des améliorations substantielles.

L'importance du rôle joué par les champignons lors de l'installation des scolytides étudiés en Amérique du Nord conduit naturellement à développer des investigations analogues en Europe. Il importe en effet d'examiner si les champignons véhiculés par les espèces européennes jouent un rôle semblable à celui de leurs homologues américains. Jusqu’à présent seul Ips typographus sur épicéa en Finlande a fait l'objet de recherches à ce sujet (Christiansen \& HornTvedt, 1983 ; Horntvedt et coll., 1983) ; le processus d'installation apparaît pour lui analogue à celui décrit aux Etats-Unis. Des études doivent être développées pour les autres espèces. Les champignons associés à Ips acuminatus et Ips sexdentatus sont déjà connus de Suède ou d'Allemagne (RENNERFELd, 1950 ; MATHIESEN-KäÄrIK, 1953, 1960 ; FranCKE-Grossman, 1967) mais leur présence chez $T$. piniperda, contrairement au cas de Tomicus minor. paraît très incertaine tandis que $D$. micans ne semble pas en posséder. Le cas de ce dernier, chez lequel on ne connaît pas non plus de phéromones d'agrégation à l'état adulte, doit sans doute être abordé de façon différente de celui des autres espèces. Par ailleurs, même si le rôle des champignons apparaît important dans les mécanismes d'installation, il ne doit pas faire sous-estimer la part des stimuli gustatifs.

\subsection{Les niveaux de population}

Comme celle de la vigueur des arbres, leur connaissance est une condition nécessaire pour estimer les risques de dégâts. Des études sur la dispersion et la répartition des insectes en phase endémique sont nécessaires mais, comme on l'a déjà signalé, il conviendra d'aborder le problème de manière différente selon l'espèce considérée (Lieutier, 1984). Ainsi, le cas des Ips qui peuvent posséder parfois jusqu'à trois générations annuelles et qui se déplacent en volant, ne pourra être considéré de la même façon que celui de $D$. micans, pour lequel le cycle peut s'étendre sur plusieurs années et dont le mode de déplacement est très mal connu. Dans le cas de $T$. piniperda, le problème est encore d'une autre nature car l'insecte occupe trois types de localisation (le tronc, les pousses, la base des arbres) au cours d'une même génération et peut se déplacer pendant sa maturation dans les pousses. Pour cet insecte, il serait très utile de déterminer la liaison spatiale entre les foyers de reproduction sur tronc et les attaques sur pousses, ainsi qu'entre les sites d'hibcrnation et les sites de reproduction. 
La mise au point d'une méthode d'échantillonnage devrait ensuite être tentée. Celle-ci s'avère évidemment très délicate avec les $I p s$ et $D$. micans par suite de la répartition discontinue de ces insectes à l'échelle de la parcelle (localisation uniquement au niveau des troncs). Toutefois, $T$. piniperda, pendant sa phase de maturation dans les pousses et par suite de la chute de celles-ci au sol, pourrait constituer un bon modèle de départ pour cet échantillonnage. Il conviendrait alors d'établir des indices d'abondance dont on connaîtrait la correspondance avec le niveau réel des populations. Ces indices pourraient être des nombres de captures par exemple (pièges à phéromones, pièges gluants ou autres types de pièges). Dans la mise en place du dispositif d'échantillonnage et dans la constitution des indices, il conviendrait aussi, évidemment, de tenir comple de l'hétérogénéité parcellaire. Ultérieurement, pourrait alors être abordée l'étude des facteurs responsables des variations des niveaux de populations c'est-à-dire la dynamique de population sensu-stricto. Ces études sont assez avancées aux Etats-Unis (Coulson, 1979 ; Berryman, 1982 b) mais nécessitent pour les espèces françaises la connaissance préalable d'un certain nombre de facteurs démographiques fondamentaux. Le plus important d'entre eux est, sans doute, la relation existant entre la densité d'attaque, le taux de réémergence des femelles après leur pontc, et leur fécondité. La productivité en scolytes des arbres attaqués en fonction de différents facteurs tels que épaisseur du liber, taille des arbres, épaisseur d'écorce, etc. devra aussi être appréciée, en tenant compte de l'existence de la relation précédente.

\section{Conclusion}

Contrairement aux recherches sur les phéromones d'agrégation, les études menées aux Etats-Unis sur les réactions de défense des arbres ont abouti rapidement à l'établissement d'un modèle cohérent intégrant l'ensemble des données actuelles sur les espèces concernées, et expliquant à la fois le mécanisme d'installation des scolytes sur les arbres et la dynamique de leurs populations. Ces résultats intéressants ne sont sans doute pas sans rapport avec une conception différente des relations plantesinsectes. Les études sur les phéromones n'ont, le plus souvent, considéré l'arbre que comme un support ayant une composition chimique définie; ce faisant, elles ont négligé qu'il s'agissait d'un être vivant, capable de réagir aux agressions, avec la variabilité que comporte toute population naturelle. Cette deuxième conception était beaucoup plus juste; elle s'est révélée aussi beaucoup plus profitable.

Les recherches en France ont, certes, un retard important dans ces domaines mais elles devraient être fructueuses, à condition que l'on sache attribuer à l'arbre isa place et son rôle exact et que l'on sache aussi tirer la leçon des expériences américaines.

Reçu en décembre 1984.

Acceplé en février 1985. 


\title{
Summary
}

\section{Bark beetles - host interactions : importance and research prospects}

\begin{abstract}
Bark beetles damages in the forest coniferous stands arise from two complementary factors, on one hand the existence of weakened trees owing to a stress, on the other hand a certain level of pest populations. A rapid presentation of the present state of the researches in this field is carried out, especially from the north american works and some european ones, emphasizing on those which take into account the defense reactions of the trees. Some research trends are then proposed for France. The general suggested proceeding in short-range, is to appreciate in what extent and for what species the "american model » is transposable in France; as a matter of fact, some european bark beetles of economic importance have a systematic situation and a biology rather different from the north american species which contributed to the construction of the model. In longer-range, the understanding of the procedures of establishment of the french species might be aimed, with intent to propose some complementary criterion for the selection of the trees; the development of a population level rating might also be aimed. The suggested research prospects are : detailed study of the physiological disturbances owing to the water stress : development of tests to appreciate the state of deficiency of the trecs; role of associated fungi in the establishment of the French bark beetles species; dispersal and distribution of the insects in an endemic stage ; development of sampling procedures at the stand level-study of the factors involved in the variations of the populations levels.
\end{abstract}

\section{Références bibliographiques}

Amman G.D., 1972. Mountain pine beetle brood production in relation to thickness of lodgepole pine phloem. J. econ. entomol., 65, 138-140.

Berryman A.A., 1972. Resistance of conifers to invasion by bark beetle fungus associations. Bio. Science, 22, 599-601.

Berryman A.A., 1976. Theoretical explanation of mountain pine beetle dynamics in lodgepole pine forests. Envir. entomol., 5, 1225-1233.

Berryman A.A., 1978. Towards a theory of insect epidemiology. Res. pop. ecol., 19, 181-196. BerRyman A.A., 1982 a. Biological control, thresholds, and pest outbreaks. Environ. entomol., 11, 544-549.

Berryman A.A., 1982 b. Population dynamics of bark beetles. In : Mitron J.B., Sturgeon K.B. «Bark beetles in north american conifers». Univ. Texas Press, Austin, 264-314.

Brand J.M., Bracke J.W., Markovetz A.J., Wood D.L., Browne L.F., 1975. Production of verbenol pheromone by a bacterium isolated from bark beetles. Nature, 254, 136-137.

Carle P., 1975. Problèmes posés par les ravageurs xylophages des conifères en forêt méditerranéenne. Rev. For. Fr., 27, 283-296.

Chararas C., 1959 a. L'attractivité exercée par les conifères à l'égard des Scolytidae et le rôle des substances terpéniques extraites des oléorésines. Rev. pathol. vég. entomol. agric. Fr., 38, 113-129.

Chararas C, 1959 b. Les variations de la pression osmotique des conifères, facteurs déterminant la pénétration des Scolytidae (insectes; coléoptères). C.R. Acad. Sci., 248, 1407-1410.

Chararas C., 1962. Etude biologique des scolytides des conifères. Lechevallier éd., Paris, $556 \mathrm{p}$.

Chararas C., 1974. La pression osmotique des essences forestières et ses rapports avec l'installation des insectes xylophages. In : PESSON P., «Ecologie forestière». GauthierVillars, 193-218. 
Chararas C., 1979. Ecophysiologie des insectes parasites des forêts. Edité par l'auteur, Paris, 298 p.

Chararas C., Riviere J., Ducauze C., Rutledge D., Delpui G., Cazelles M.T., 1980. Bioconversion d'un composé terpénique sous l'action d'une bactérie du tube digestif de Phloeosinus armatus (coléoptère; Scolytidae). C.R. Acad. Sci. Paris, 291, 299-302.

Christiansen E., Hornt vedt R., 1983. Combined Ips/Ceratocystis attack on Norway spruce, and defensive mechanisms of the trees. $Z$. ang. entomol., 96, 110-118.

Coulson R.N., 1979. Population dynamics of bark beetle. Ann. Rev. entomol, 24, 417-447.

Francke-GrosmanN H., 1967. Ectosymbiosis in Wood-inhabiting insects. In : «Symbiosis», 2, S.M. Henry Ed. Acad. Press, New York, 141-205.

Graham K., 1967. Fungal-insect mutualism in trees and timber. Ann. Rev. entomol., 12, $105-126$.

Hanover J.W., 1975. Physiology of tree resistance to insects. Ann. Rev. entomol., 20, 75-95.

Hodges J.D., LoRı P.L., 1969. Carbohydrate and nitrogen fractions of the inner bark of loblolly pines under moisture stress. Can. J. Bot., 47, 1651-1657.

Hodges J.D., LorJo P.L., 1971. Comparison of field techniques for measuring moisture stress in large loblolly pines. Forest Science, 17, 220-223.

Horntvedt R., Christiansen E., Solheim H., Shasheng W., 1983. Artificial inoculation with Ips typographus associated blue-stain fungi can kill healthy Norway spruce trees. Meddelelser fra Norsk Institut for Skogforskining, 38, 1-20.

Hynum B.G., Berryman A.A., Dendroctonus ponderosae (Coleoptera : Scolytidae) preaggregation landing and gallery initiation on lodgepole pine. Can. Entomol., 112, 185-191.

Klement Z., Goodman R.N., 1967. The hypersensitive reaction to infection by bacterial plant pathogens. Ann. Rev. Phytopathol., 5, 17-44.

Lieutier F., 1984. Impact économique des scolytides : voies de recherches. C.R. Acad. Agric. Fr., 70, 835-843.

Mathiesen-KaARIK A., 1953. Eine Übersich über die gewöhnlichen mit Borkenkäfern assocïerten Bläuepilze in Schweden. Medd. statens Skogsfössökanst., 43, 1-74.

Mathiesen-KaARiK A., 1960. Studies on the ecology, taxonomy and physiology of swedish insect-associated blue stain fungi, especially the genus Ceratocystis. Oikos., 11, 1-25.

MOEK H.A., WOOD D.L., LindaHL K.Q., 1981. Host selection behavior of bark beetles (Coleoptera : Scolytidae) attacking Pinus ponderosa, with special emphasis on the western pine beetle, Dendroctonus brevicomis. J. chem. ecol., 7, 49-83.

Pesson P., Chararas C., 1969. Les scolytides, insectes ravageurs mondiaux des forêts de conifères. L'année biol., 8, 683-733.

RaFfa K.F., Berryman A.A., 1980. Flight response and host selection by bark beetles. Proc. Ind I.U.F.R.O. Conf. on "dispersal of forest insects : Evaluation, Theory and management $\gg$. Berryman A.A., SafranyiK L. (Ed.), 213-233.

RAFFA K.F., BerRyman A.A., 1982 a. Gustatory cues in the orientation of Dendroctonus ponderosue (Coleoptera : Scolytidae) to host trees. Can. entomol., 114, 97-104.

Rafis K.F., Berryman A.A., 1982 b. Accumulation of monoterpenes and associated volatiles following inoculation of grand fir with a fungus transmitted by the fir engraver, Scolytus ventralis (Coleoptera : Scolytidae). Can. Entomol., 114, 797-810.

RaFFa K.F., Berryman A.A., $1982 \mathrm{c}$. Physiological differences between lodgepole pines resistant and susceptible to the moutain pine beetle and associated microrganisms. Environ. entomol., 2, 486-492.

RafFA K.F., Berryman A.A., 1983 a. Physiological aspects of lodgepole pine wound responses to a fungal symbiont of the mountain pine beetle, Dendroctonus ponderosae (Coleoptera : Scolytidae). Can. Entomol., 115, 723-734.

RaFfa K.F., Berryman A.A., 1983 b. The role of host resistance in the colonization behavior and ecology of bark beetles (Coleoptera : Scolytidae). Ecol. monog., 53, 27-49.

Reid R.W., Wiitney H.S., Warson J.A., 1967. Reactions of lodgepole pine to attacks by Dendroctonus ponderosae Hopkins and blue stain fungi. Can. J. Bot., 45, I115-1126.

RENNERIELD E., 1950. Über den Zusammenhang zwischen dem Verblauen des Holzes und den Insekten. Oikos, 2, 120-137. 
Russei, C.E., Berryman A.A., 1976. Host resistance to the fir engraver beetle. 1. Monoterpene composition of Abies grandis pitch blisters and fungus-infected wounds. Can. J. Bot.. 54, 14-18.

Safranyk L., Shrimpton D.M., Whitney H.S., 1975. An interpretation of the interaction between lodgepole pine, the mountain pine bectle, and its associated blue stain fungi in western Canada. In : «Management of Lodgepole Pine ecosystems». D.M. BaumgarTner Ed., Pullman, Wash state univ. Coop. Ext. Serv., 406-428.

SchonhERR J., 1977, Importance of visual stimuli in the host sclection behavior of bark bectles (Dentroctonus ponderosae and Ips montana). Coll. Int. C.N.R.S. "Comportement des insectes et milieu trophique 》, 265, 187-193.

Shrimpton D.M., 1973. Extractives associated with the wound response of lodgepole pine beetle and associated micro-organisms. Can. J. Bot., 51, 527-534.

Shrimpton D.M., 1978. Resistance of lodgepole pine to moutain pine bectle infestation. In : Berryman A.A., Amman G.D., Stark R.W., Kibbee D.L. (Eds). Theory and practice of mountain pine beetle management in lodgepole pine forests. College of for. Res. Univ. Idaho. Moscow, 64-76.

Shrimpton D.M., Reid R.W., 1973. Change in resistance of lodgepole pine to mountain pine beetle between 1965 and 1972. Cam. J. For. Res., 3, 430-432.

Silverstein R.M., 1977. Complexity, diversity and specificity of behavior modifying chemicals : Examples mainly from Coleoptera and Hymenoptera. In : SHOREY J.J., Mc Kelvey Jr. (Eds). "Chemical control of insect behavior : theory and application》, N.Y. Wiley, 2.31-251.

VITE J.P., 1961. The influence of water supply on olcoresin exudation pressure and resistance to bark beetle attack in Pinus ponderosa. Contrib. Boyce. Thomp. Inst., 21, 37-66.

VITE J.P., WoOD D.L., 1961. A study on the applicability of the measurement of oleoresin exudation pressure in determining susceptibility of second growth Ponderosa pine to bark beetles infestation. Contrib. Boyce. Thomp. Litst., 21, 67-78.

Waring R.H., Pitman G.B., 1980. A simple model of host resistance to bark beetles. Forest research laboratory, Research notes, 65, $2 \mathrm{p}$.

Waring R.H., Pitman G.B., 1983. Physiological stress in lodgepole pine as a precursor for mountain pinc beetle attack. $Z$. angew. entomol., 96, 265-270.

Wong B.L., Berryman A.A., 1977. Host resistance to the fir engraver beetle. 3. Lesion development and containment of infestation by resistant Abies grandis inoculated with Trichosporium symbioticum. Can. J. Bot., 55, 2358-2365.

Wood D.L., 1982. The role of pheromones, kairomones and allomones in the host selection and colonization behavior of hark beetles. Am. rev. entomol., 27, 411-446.

Wrigiht L.C., Berryman A.A., Gurusiddain H.S., 1979. Host resistance to the fir engraver bectle, Scolytus ventralis (Colcoplera - Scolytidae). 4. Effect of defoliation on wound monoterpene and inner bark carbohydrate concentrations. Can. Entomol., III, 1255-1262. 